\title{
Can lenalidomide be accused to induce high grade pattern of fever in a multiple myeloma patient? A Question to be answered
}

\author{
Ghada EL Gohary ${ }^{1,2 *}$, Mohammad Al Nahedh ${ }^{3}$ and Riad El Fakih ${ }^{4}$ \\ ${ }^{1}$ Department of Adult Hematology/Oncology, King Khaled University Hospitals, College of Medicine, King Saud University, Riyadh, Saudi Arabia \\ ${ }^{2}$ Department of Adult Hematology/Internal Medicine, Ain Shams University Hospitals, College of Medicine, Cairo, Egypt \\ ${ }^{3}$ Oncology Pharmacy Services, King Faisal Specialist Hospital and Research Centre, Riyadh, Kingdom of SaudiArabia \\ ${ }^{4}$ Section of Adult Hematology/HCT Oncology Centre, King Faisal Specialist Hospital and Research Centre, Riyadh, Kingdom of Saudi Arabia
}

\begin{abstract}
Lenalidomide is a second-generation immunomodulatory agent and a potent analogue of thalidomide that is FDA approved mainly for the treatment of multiple myeloma (MM) and transfusion-dependent anaemia due to low or intermediate-1- risk myelodysplastic syndromes (MDS) associated with 5q deletion among other indications. Through its action on the immune system, lenalidomide alters the production of different cytokines ultimately resulting in immune activation against tumours. This immune activation may lead to collateral immune toxicities like fever, angioedema, Stevens-Johnson syndrome, tumour flare and others. Here we report a case of lenalidomide-induced high grade fever in a patient with MM and we summarize the literature about the physiology of such reaction and how to mitigate this adverse event.
\end{abstract}

\section{Case report}

A 59 year-old-woman with newly diagnosed IgG kappa MM presented to the emergency department with fever and generalized body aches one day after starting her first cycle of therapy with lenalidomide, bortezomib and dexamethasone. She denied any shortness of breath, cough, runny nose, dysuria, diarrhoea, abdominal pain, and headache or neck stiffness. Physical examination was unremarkable except for fever (temperature of $39^{\circ} \mathrm{C}$ ) and tachycardia. Patient was started on broad spectrum antibiotics and admitted for workup. Infectious workup included repeated blood and urine cultures, chest x-ray, nasal swab for viruses and all were negative. Her complete blood count and differential was within normal limits. Patient remained febrile for 5 days (Figure 1), despite escalating and changing her antimicrobial therapy. After six days of hospitalization, antibiotics and lenalidomide were discontinued. Fever subsided within few hours and patient was then discharged in stable condition. The patient was then treated with cyclophosphamide, bortezomib and dexamethasone and underwent autologous hematopoietic cell transplant after achieving a very good partial remission. The patient refused maintenance therapy posttransplant and relapsed 2 years after transplant. Upon relapse, patient was treated with low dose lenalidomide ( $10 \mathrm{mg}$ daily), bortezomib and dexamethasone and tolerated this regimen well and later lenalidomide dose was escalated to the standard dose of $25 \mathrm{mg}$ daily with good tolerance.

\section{Discussion}

Lenalidomide is a 4-amino-glutamyl analogue of thalidomide, modified in a way to minimize the neurologic side effects and to improve the efficacy of thalidomide. It is a second-generation immunomodulatory agent approved by FDA for clinical use mainly in myelodysplastic syndromes with deletion of chromosome $5 \mathrm{q}$, multiple myeloma among other cancers [1]. As an immunomodulatory drug it affects both the cellular ( $\mathrm{T}$ and NK cells activation) and humoral components of the immune system, which results in down regulation (TNF- $\alpha$, IL-6, IL-8) or up regulation (IL-2 and IFN- $\gamma$ ) of various cytokines and growth factors and eventually leads to changes in the tumour microenvironment [2]. Fever is frequently encountered while caring for patients with hematologic malignancies. Lenalidomide induced fever is not infrequent and may be easily overlooked because it is not appropriately reported or highlighted in the literature.

Cancer patients may develop fever for multiple reasons (infections, thrombosis, drugs...). Drug fever is a febrile response that coincides temporally with the administration of a drug and disappears after discontinuation of the offending agent. It is usually suspected when no other causes of fever can be identified, often after antimicrobials have already been started. Fever can be the sole manifestation of an adverse drug reaction in 3-5\% of cases [3,4]. The mechanisms of drug fever are multiple and, in many cases, are poorly or incompletely understood $[3,4]$. The lenalidomide package insert ranks fever as one of the most common adverse reactions that can affect more than $20 \%$ of the patients on lenalidomide. The mechanism of lenalidomide-induced fever remains unclear however it is well known that lenalidomide can increase the production of pro-inflammatory cytokines (IL-6 and TNF- $\alpha$ ) that cause elevation of CRP [5], enhances immunological synapse formation and upregulates CD80 on tumor cells which leads to

*Correspondence to: Ghada EL Gohary, Department of Adult Hematology/ Oncology, King Khaled University Hospitals, College of Medicine, King Saud University, Riyadh, Saudi Arabia, Tel: +966 11467 0000; E-mail: ghelgohary@ gmail.com,gelgohary@ksu.edu.sa

Key words: lenalidomide, fever, toxicity

Received: February 20, 2019; Accepted: March 08, 2019; Published: March 12, 2019 


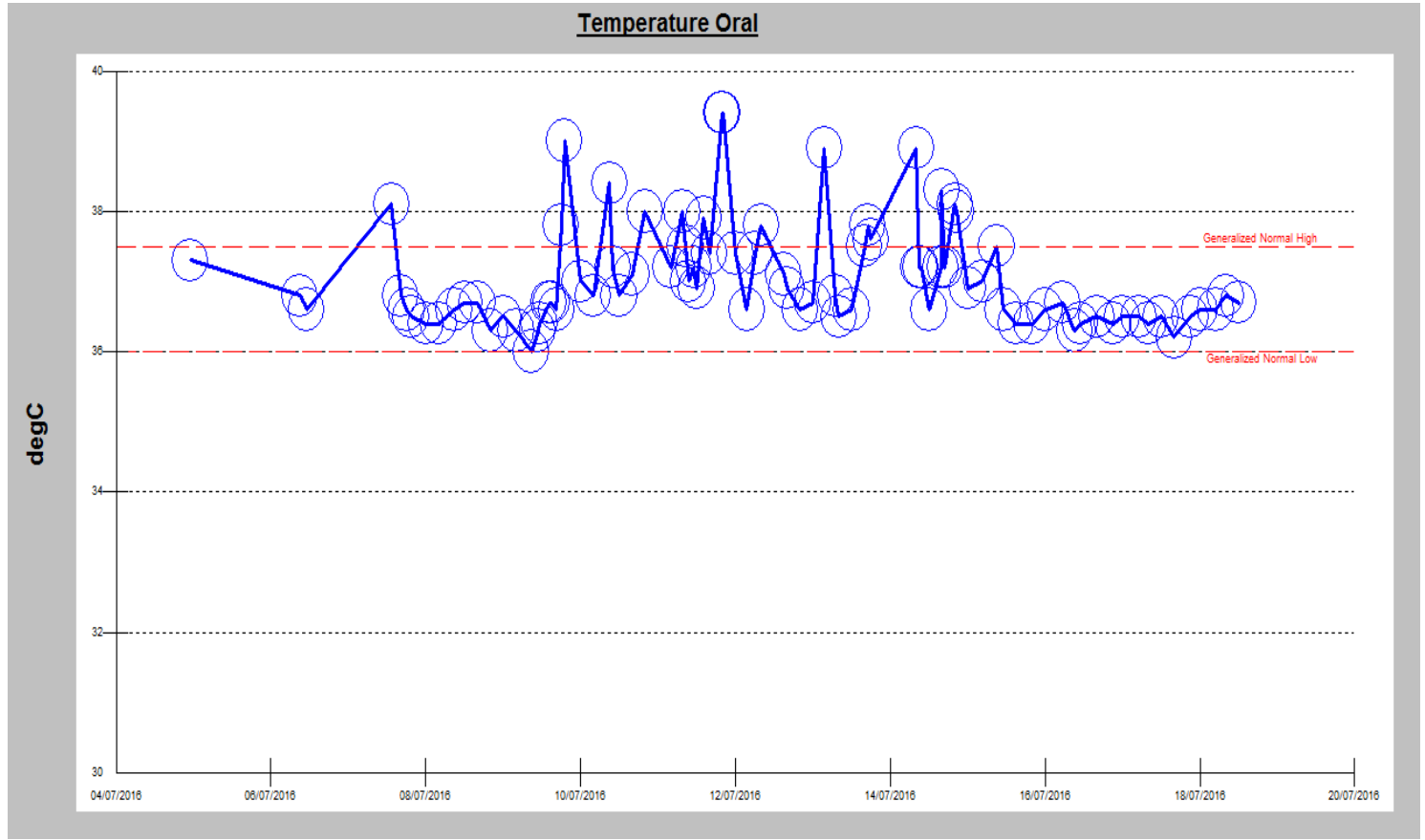

Figure 1. Physical examination of temperature

T cell activation and rapid cytokine release [6,7], and these in turn may cause fever. In a published case report a patient with MM was started on lenalidomide and 4 days later he was admitted with high grade fever and elevated CRP, his pre-treatment plasma interleukin-6 and tumor necrosis factor levels were normal and these increased significantly after starting lenalidomide. The fever resolved spontaneously after stopping lenalidomide [7]. Tumor flares have been reported after using lenalidomide for the treatment of chronic lymphocytic leukemia [8], and this might be another mechanism of lenalidomide induced fever, however the exact mechanism remains unclear [9]. The management of these febrile reactions consists of holding the medication until resolution of the symptoms; patient can be re-challenged at a lower dose followed by escalation as tolerated.

\section{Conclusion}

In summary, fever due to lenalidomide therapy seems to be frequent but not highlighted in the medical literature. Fever is not reported with the use of thalidomide, the first generation immunomodulatory; however, fever incidence in the phase III registration trial for pomalidomide was $27 \%$ [10]. As such this side effect might be a class side effect related to the potency of the different generations. Whether the incidence of fever correlates with the activity of these agents remains unanswered, keeping in mind that neither the degree of $\mathrm{T}$ cell activation in vitro nor the severity of the cytokine release syndrome correlated with the activity of lenalidomide in lymphomas [11]. Medical teams might consider holding the medication without rushing to costly investigations and without starting antibiotherapy especially in the absence of infectious symptoms. Physicians should be aware of this potential association given the increased frequency of lenalidomide usage. Further studies are needed to clarify whether this phenomenon influences the clinical outcomes.

\section{References}

1. Kotla V, Goel S, Nischal S, Heuck C, Vivek K, et al. (2009) Mechanism of action of lenalidomide in hematological malignancies. J Hematol Oncol 2: 36. [Crossref]
2. Chanan-Khan AA, Cheson BD (2008) Lenalidomide for the treatment of B-cell malignancies. J Clin Oncol 26: 1544-1552. [Crossref]

3. Thong BY, Tan TC (2011) Epidemiology and risk factors for drug allergy. Br J Clin Pharmacol 71: 684-700. [Crossref]

4. Riedl MA, Casillas AM (2003) Adverse drug reactions: types and treatment options Am Fam Physician 68: 1781-1790. [Crossref]

5. Ferrajoli A, Lee B-N, Schlette EJ, O’Brien SM, Gao H, et al. (2008) Lenalidomide induces complete and partial remissions in patients with relapsed and refractory chronic lymphocytic leukemia. Blood 111: 5291-5297. [Crossref]

6. Quach H, Ritchie D, Stewart AK, Neeson P, Harrison S, et al. (2010) Mechanism of action of immunomodulatory drugs (IMiDS) in multiple myeloma. Leukemia 24: 22. [Crossref]

7. Nakamura N, Kanemura N, Shibata Y, Matsumoto T, Mabuchi R, et al. (2014) Lenalidomide-induced cytokine release syndrome in a patient with multiple myeloma. Leuk Lymphoma 55: 1691-1693. [Crossref]

8. Moutouh-de Parseval LA, Weiss L, DeLap RJ, Knight RD, Zeldis JB (2007) Tumor lysis syndrome/tumor flare reaction in lenalidomide-treated chronic lymphocytic leukemia. J Clin Oncol 25: 5047-5047. [Crossref]

9. Morita Y, Shimada T, Yamaguchi T, Rai S, Hirase C, et al. (2011) Cytokine profiles in relapsed multiple myeloma patients undergoing febrile reactions to lenalidomide. Int $J$ Hematol 94: 583-584. [Crossref]

10. San Miguel J, Weisel K, Moreau P, Lacy M, Song K, et al. (2013) Pomalidomide plus low-dose dexamethasone versus high-dose dexamethasone alone for patients with relapsed and refractory multiple myeloma (MM-003): a randomised, open-label, phase 3 trial. Lancet Oncol 14: 1055-1066. [Crossref]

11. Wiernik PH, Lossos IS, Tuscano JM, Justice G, Vose JM, et al. (2008) Lenalidomide monotherapy in relapsed or refractory aggressive non-Hodgkin's lymphoma. J Clin Oncol 26: 4952-4957. [Crossref]

Copyright: (C2019 Gohary GEL. This is an open-access article distributed under the terms of the Creative Commons Attribution License, which permits unrestricted use, distribution, and reproduction in any medium, provided the original author and source are credited. 